\title{
ARTICLE OPEN Morphological instability of aqueous dissolution of silicate glasses and minerals
}

\author{
Yifeng Wang ${ }^{1}$, Carlos F. Jove-Colon ${ }^{1}$, Christoph Lenting $\mathbb{D}^{2}$, Jonathan Icenhower ${ }^{1}$ and Kristopher L. Kuhlman (D)
}

Understanding of aqueous dissolution of silicate glasses and minerals is of great importance to both Earth and materials sciences. Silicate dissolution exhibits complex temporal evolution and rich pattern formations. Recently, we showed how observed complexity could emerge from a simple self-organizational mechanism: dissolution of the silica framework in a material could be catalyzed by the cations released from the reaction itself. This mechanism enables us to systematically predict many key features of a silicate dissolution process including the occurrence of a sharp corrosion front (vs. a leached surface layer), oscillatory dissolution and multiple stages of the alteration process (e.g., an alteration rate resumption at a late stage of glass dissolution). Here, through a linear stability analysis, we show that the same mechanism can also lead to morphological instability of an alteration front, which, in combination with oscillatory dissolution, can potentially lead to a whole suite of patterning phenomena, as observed on archaeological glass samples as well as in laboratory experiments, including wavy dissolution fronts, growth rings, incoherent bandings of alteration products, and corrosion pitting. The result thus further demonstrates the importance of the proposed selfaccelerating mechanism in silicate material degradation.

npj Materials Degradation (2018)2:27; doi:10.1038/s41529-018-0047-0

\section{INTRODUCTION}

Chemical weathering of silicate minerals and glasses - the most dominant component in the Earth crust - plays a critical role in global biogeochemical cycles and directly regulates the long-term climate evolution in our Earth system. ${ }^{1-3}$ Silicate dissolution and the induced carbonate precipitation have been investigated as a means for subsurface carbon sequestration. ${ }^{4} \mathrm{~A}$ recent field test where $\mathrm{CO}_{2}$ was injected into a basaltic rock suggested that this carbonation process could happen far faster $(<2$ years) than previously postulated, ${ }^{5}$ although the underlying mechanism is still unknown. Silicate materials have also been used in numerous industrial and technological applications such as molecular sieves for chemical separation, ${ }^{6}$ catalysts for chemical conversion, ${ }^{6}$ optical fibers for communication, ${ }^{7}$ biomedical devices, ${ }^{8}$ construction materials, ${ }^{9}$ and waste forms for nuclear waste disposal. ${ }^{10,11}$ In many of these applications, the chemical durability of a material in the presence of liquid water or moisture directly determines the lifetime of the material in service. Therefore, understanding chemical alteration of silicate materials in aqueous solutions is of great importance to both Earth and materials sciences. Such an understanding is a prerequisite for a performance assessment of a silicate material as a waste form for nuclear waste disposal over a regulatory time period of up to hundreds of thousand years, ${ }^{10,11}$ a time scale beyond any possible direct experimental test.

The underlying mechanism for silicate material degradation remains controversial. Aqueous dissolution of a silicate material was proposed to start with the formation of a silica-rich surface layer on a dissolution surface in which alkali and alkaline cations are partially leached out and replaced by hydrogen ions. ${ }^{12-14}$ Recent experimental work suggests that, in a solution saturated with amorphous silica, the surface layer could form through a local structural arrangement involving little or no dissolution of silicate framework. ${ }^{15}$ The surface layer may continuously be subjected to in-situ silicate network repolymerization and reorganization, leading to the formation of a dense silica gel layer that may passivate a dissolving solid surface, resulting in a dramatic reduction in the dissolution rate. ${ }^{16}$ The traditional surface layer concept was challenged by recent observations of the existence of an extremely sharp interface between altered rims and pristine material domains, suggesting that material corrosion might be a direct dissolution-precipitation process. ${ }^{17-20}$ Oscillatory zonings, commonly observed in altered rims, seem also difficult to reconcile with the traditional surface-layer concept. ${ }^{17}$ However, the occurrence of a sharp interface is apparently not "universal"; as a matter of fact, in some cases, a gradient region ( $500 \mathrm{~nm}$ thick) has been observed between altered rims and pristine material domains. ${ }^{15}$ The contradictory observations indicate the complexity of silicate material dissolution and call for a new theory to account for such complexity. Recently, we have shown how complex behaviors observed can emerge from a simple positive feedback between dissolution-induced cation release and cationenhanced dissolution kinetics. ${ }^{21}$ This self-accelerating mechanism enables us to systematically predict the occurrence of sharp dissolution fronts (vs. leached surface layers), oscillatory dissolution behaviors, and multiple stages of glass dissolution (e.g., an alteration rate resumption at a late stage of a corrosion process).

Here we further show that the same mechanism can also lead to a morphological instability of an alteration front. Morphological instability refers to an evolution of an initially planar dissolution or growth surface into a wavy or even fingered front through its own reaction-transport dynamics. ${ }^{22}$ This instability, in combination with oscillatory dissolution predicted by our previous work, ${ }^{21}$ can

\footnotetext{
${ }^{1}$ Sandia National Laboratories, P. O Box 5800, Albuquerque, New Mexico 87185-0779, USA and ${ }^{2}$ Steinmann Institut für Geologie, Mineralogie und Paläontologie, University of Bonn, Poppelsdorfer Schloss, Bonn 53115, Germany

Correspondence: Yifeng Wang (ywang@sandia.gov)
}

Received: 8 February 2018 Revised: 1 August 2018 Accepted: 3 August 2018

Published online: 04 September 2018 
potentially give rise to a whole suite of patterning phenomena, as observed on archaeological glass samples as well as in laboratory experiments, including wavy dissolution fronts, corrosion pits, growth rings, and incoherent bandings of alteration zones. The result thus further demonstrates the importance of the proposed self-accelerating mechanism in silicate material degradation.

\section{RESULTS}

Morphological instability and the underlying mechanism

Many excellent observations have been made on the structures and pattern formations of altered silicate materials. Dohmen et al. performed a detailed backscattered electron imaging study on two borosilicate glasses. ${ }^{23}$ They showed that among 16 dissolution experiments conducted, except one with an initial $\mathrm{pH}$ of about zero, all other experiments produced distinct lamellar layers with a thickness of $5-100 \mu \mathrm{m}$. Some of the layers form a coherent (i.e., parallel) wavy front with the convex side pointing toward a pristine glass domain. The wavy front seems to initiate from earlier-developed dissolution pits on a dissolution surface. Extensive pitting on a dissolution surface was observed on some samples (ref. ${ }^{23}$, fig. 5). A gap seems to exist between an altered rim and a neighboring pristine glass domain, indicating an atomically sharp reaction interface. In addition to the coherent lamellar layers, incoherent wavy layers were also observed, in which two neighboring layers are not parallel (ref. ${ }^{23}$, figs. $\left.3 c, g\right)$. In some occasions, hemispherical-shape incoherent layers penetrate deeply from a fracture surface into a pristine glass domain. Similar structural patterns were also observed on archaeological glass samples. ${ }^{24,25}$ On those samples, Silvestri et al. showed that within each individual wavy lamellar layer there occur spongy, distorted and branched structures (ref. ${ }^{24}$, fig. 3), indicating complex dissolution and precipitation involved in the formation of each individual layer. Concentric growth rings were also observed on a view plane parallel to a material dissolution surface (ref. ${ }^{25}$, fig. $7 \mathrm{~b}$ ). Some of typical patterning phenomena in silicate glass alteration are shown in Fig. 1.

We now want to show how these observed complex patterning phenomena in silicate material degradation can emerge from a morphological instability of a dissolution surface through the same mechanism we proposed earlier for oscillatory dissolution of silicate materials: the dissolution of a silicate material can potentially be catalyzed by the cations released from the reaction itself. ${ }^{21}$ This mechanism was postulated from the observed Vshape dependence of a far-from-equilibrium silicate material dissolution rate on solution $\mathrm{pH}^{21,26-29}$ This mechanism operates on the right branch of the rate curve in Fig. 2. Since the V-shaped $\mathrm{pH}$ dependence of the dissolution rate exists for a diverse set of silicate minerals including quartz, ${ }^{21,26-29}$ this dependence must be related to the dissolution of silica framework-a common structural component in all these minerals. In addition to the catalytic effect of hydroxyls, Rimstidt recently showed that a cation itself such as $\mathrm{Na}^{+}$could also catalyze quartz dissolution. ${ }^{30}$ As elaborated previously, ${ }^{21}$ this self-accelerating mechanism can systematically account for the occurrence of multiple stages (or regimes) of silicate material degradation. For an illustration, let's assume that the material degradation starts in an acid solution (on the left branch of the dissolution curve in Fig. 2), under which the dissolution rate of silica framework is higher than that for cation leaching, and as a result the material would dissolve congruently with no leached layer developed. As the $\mathrm{pH}$ of the solution increases due to the accumulation of the leached cations, the dissolution rate becomes lower than the leaching rate, leading to the formation a leached surface layer. As the $\mathrm{pH}$ continuously rises, the system moves from the left branch to the right branch of the dissolution curve. When the dissolution rate becomes on the same order of magnitude as the mass exchange rate with the bulk solution, oscillatory dissolution may emerge. As the $\mathrm{pH}$ of the solution further increases, the dissolution rate eventually overtakes the mass exchange rate, leading to a "runaway" situation with a sharp increase in the cation concentration at the interface as well as in the dissolution rate. The sharp increase in both cation concentration and $\mathrm{pH}$ inevitably causes zeolite precipitation. The whole dissolution process illustrated in Fig. 2 would also be influenced by the dissolved silica concentration. As a matter of fact, it is the interplay between the cation and the silica concentrations that gives rise to oscillatory dissolution of silicate materials. $^{21}$

An actual precipitation process of alteration products could be complex. Since we here focus on the morphological instability of a reaction front, for simplicity, we assume that the overall dissolution-precipitation reaction process can stoichiometrically be represented by reaction (1):

Silicate $\stackrel{\text { Cations }}{\longrightarrow}$ Alteration products $+a_{s} \mathrm{SiO}_{2}(\mathrm{aq})+a_{c}$ Cations $(\mathrm{aq})$

where $a_{s}$ and $a_{c}$ are the stoichiometric coefficients of dissolved $\mathrm{SiO}_{2}$ and cations, respectively, in the overall silicate alteration reaction. Since most of $\mathrm{SiO}_{2}$ released from the dissolution is reincorporated into the alteration products, $a_{s}$ is expected to be relatively small compared to $a_{c}$. The cations in reaction (1) refer to alkali cations (mainly $\mathrm{Na}^{+}$) and part of alkaline-earth cations that remain as dissolved cationic species in solution after their release from material degradation. Let's assume that the dissolution of a silicate material starts on a planar reaction front (Fig. 3). As the alteration reaction proceeds, the released $\mathrm{SiO}_{2}(\mathrm{aq})$ and cations accumulate at the front. In an actual dissolution process, the reaction front is inevitably subjected to environmental perturbations, resulting in small fluctuations in reaction rate on the dissolution front. Based on the self-accelerating mechanism proposed above, in a faster dissolution region (labeled "a" in Fig. $3)$, more cations would become released and accumulated, which in turn further accelerates the dissolution at this location. The opposite happens at location "b", where the cations can be relatively easier to diffuse away due to a shorter diffusion distance through the alteration zone (Fig. 3). As a result, an environmental perturbation to the initially planar front could be amplified, leading to the formation of a wavy front. As a dissolution front becomes unstable, two other factors would come into play: surface tension, which tries to minimize the surface area of the front and therefore tends to restore the initial planar front, and diffusion, which tends to smooth out the concentration fluctuations on the dissolution surface. The final wave length of a wavy front would then be determined by the interplay between the morphological instability and these countering factors.

\section{Mathematical formulation}

To further elaborate the morphological instability of a dissolution front, let's consider a modeling system as illustrated in Fig. 3. The system is divided into two physical domains: the pristine glass and the alteration zone. The dissolved species released from the alteration [reaction (1)] communicate with the bulk solution via diffusion through the corrosion products. The chemistry of the bulk solution outside the alteration zone is assumed to remain constant. Also assume that the position of the dissolution front at time $t$ can be described by $X=F(Y, t)$.

Within $F(Y, t)<X<L$, the diffusion of the dissolved species across the alteration zone can be described by:

$\frac{\partial C_{s}}{\partial t}=D_{s} \nabla^{2} C_{s}$

$\frac{\partial C_{c}}{\partial t}=D_{c} \nabla^{2} C_{c}$ 

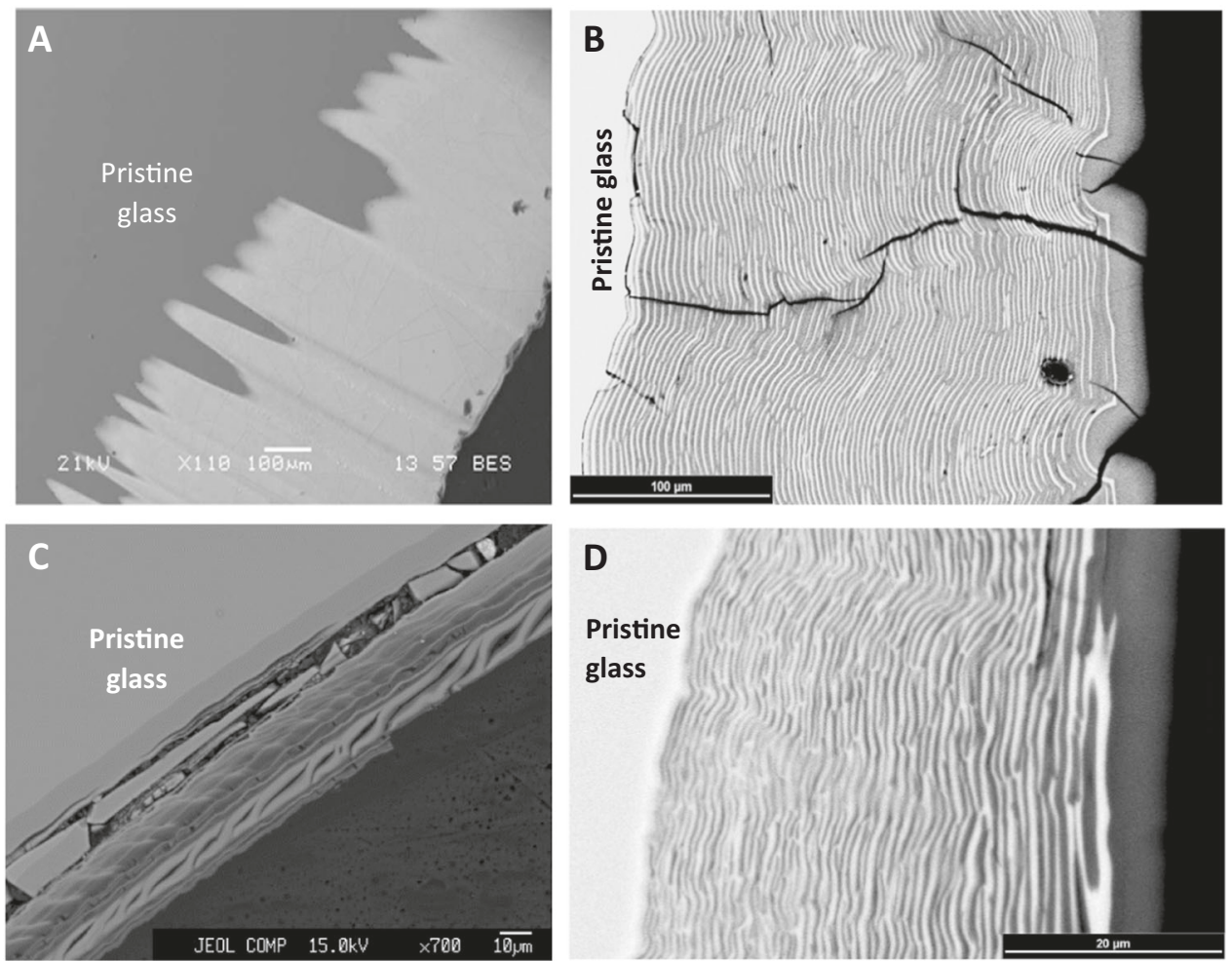

Fig. 1 Backscattered electron images of corroded glass samples. a Fingered dissolution front of an International Simple Glass (ISG) sample altered in deionized water at $90^{\circ} \mathrm{C}$ and $\mathrm{pH}_{25}{ }^{\circ} \mathrm{C}=9$ under a flow-through condition for 55 days (scale bar-100 $\mu \mathrm{m}$ ). b Coherent wavy corrosion bands (chemical pattern) observed in corroded German WAK glass from an experimental series conducted at $150^{\circ} \mathrm{C}, \mathrm{pH} \mathrm{init}, 25^{\circ} \mathrm{C}=0$, and $\mathrm{S} / \mathrm{V}=$ $0.2 \mathrm{~cm}^{-1}$ (ref. $^{35}$ ) (scale bar-100 $\mu \mathrm{m}$ ). c Incoherent banding (bifurcations) at a glass dissolution surface and incoherent structures within individual bands in U-bearing, soda-lime glass beads corroded at $90^{\circ} \mathrm{C}, \mathrm{pH}_{\text {init, } 25^{\circ} \mathrm{C}}=7.1$, and $\mathrm{S} / \mathrm{V}=0.6 \mathrm{~cm}^{-1}\left(\mathrm{ref}^{23}\right)(\mathrm{scale}$ bar- $-10 \mu \mathrm{m})$. d Incoherent banding in German WAK glass from an experimental series conducted at $150^{\circ} \mathrm{C}, \mathrm{pH}_{\text {init,2 }}{ }^{\circ} \mathrm{C}=0, \mathrm{and}^{\mathrm{S}} / \mathrm{N}=0.2 \mathrm{~cm}{ }^{-1}\left(\mathrm{ref}^{35}\right)(\mathrm{scale}$ bar-20 $\mu \mathrm{m})$

where $X$ and $Y$ are the spatial coordinates (Fig. 3); $t$ is the time; $C_{s}$ and $C_{c}$ are the concentrations of dissolved $\mathrm{SiO}_{2}$ and cations, respectively; $D_{s}$ and $D_{c}$ are the effective diffusion coefficients of the two species, respectively; and $L$ is the thickness of the alteration zone.

At the dissolution front $X=F(Y, t)$, the reaction rate, $R$, and the diffusion fluxes are related by (ref. $\left.{ }^{31}\right)$ :

$$
\begin{aligned}
& a_{s} R=-D_{s} \vec{\nabla} C_{s} \cdot \vec{n} \\
& a_{c} R=-D_{c} \vec{\nabla} C_{c} \cdot \vec{n} \\
& R=k\left[1+\beta\left(\frac{C_{c}}{C_{I E P}}\right)^{m}\right]\left(C_{s}^{e}-C_{s}\right) \\
& C_{s}^{e}=C_{s}^{e}(\infty)(1+\Gamma \mathrm{K}) \\
& \mathrm{K}=-\frac{\partial^{2} F}{\partial Y^{2}} /\left[1+\left(\frac{\partial F}{\partial Y}\right)^{2}\right]^{3 / 2} \\
& \vec{n}=\left(1,-\frac{\partial F}{\partial Y}\right)^{T} /\left[1+\left(\frac{\partial F}{\partial Y}\right)^{2}\right]^{1 / 2}
\end{aligned}
$$

where $\vec{n}$ is the normal vector of the dissolution front pointing toward the alteration zone; $k$ is the reaction rate constant; $\beta$ and $m$ are constants characterizing the catalytic effect of cations on the dissolution rate of silica framework; $C_{\text {IEP }}$ is the concentration of cations at the isoelectric point (IEP), at which the dissolution rate reach its minimum (Fig. 2); $C_{s}^{e}$ is the solubility of silica framework on a surface of curvature $K ; C_{s}^{e}(\infty)$ is the solubility of silica framework on a planar surface; $\Gamma$ is the surface tension of the interface between the pristine glass and the solution; and superscript $T$ in Eq. (9) represents the transposition of a vector. Equations (7) and (8) capture the effect of surface tension on the solubility of silica framework.

At $X=L$, we have:

$$
\begin{aligned}
& C_{s}=C_{s}^{L} \\
& C_{c}=C_{c}^{L}
\end{aligned}
$$

The kinematics of the dissolution front can be described by: ${ }^{31}$

$$
\begin{aligned}
& \rho \frac{\partial F}{\partial t}=\bar{R}-R\left[1+\left(\frac{\partial F}{\partial Y}\right)^{2}\right]^{1 / 2} \\
& \bar{R}=k\left[1+\beta\left(\frac{\bar{C}_{C}}{C_{\text {IEP }}}\right)^{m}\right]\left[C_{s}^{e}(\infty)-\bar{C}_{s}\right]
\end{aligned}
$$

where $\rho$ is the molar density of the silicate material; $\bar{R}$ is the reaction rate evaluated at the planar front; and $\bar{C}_{c}$ and $\bar{C}_{s}$ are the concentrations of dissolved $\mathrm{SiO}_{2}$ and cations at the planar front, respectively.

Linear stability analysis

Equations (2) through (13) were first scaled, using:

$$
\begin{array}{llll}
x=X / L & y=Y / L & f=F / L & \tau=t / T \\
u=C_{s} / C_{s}^{e}(\infty) & v=\frac{C_{c}}{C_{I E P}} & T=\frac{\rho L}{k C_{s}^{e}(\infty)}=\frac{L}{k} & r=\frac{T}{\rho L} R, \\
\gamma=\Gamma / L & \kappa=K L & &
\end{array}
$$




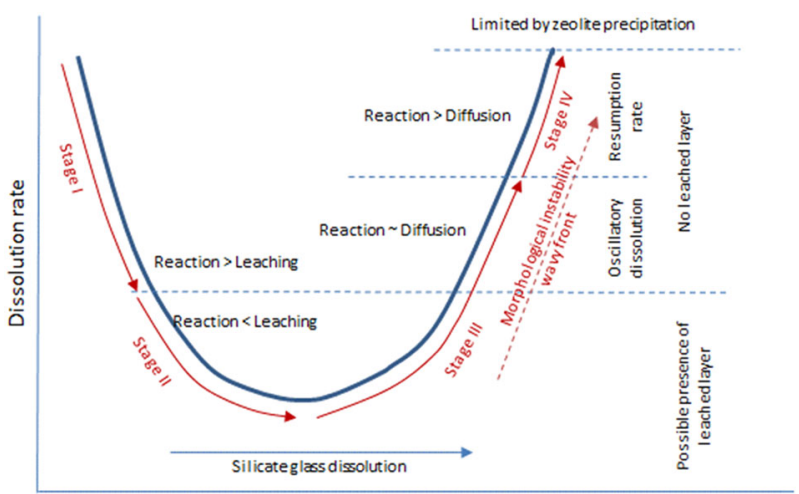

$\mathrm{pH}$ or $\log \mathrm{c}_{\mathrm{c}}$

Fig. 2 Pattern formation and transition in silicate material degradation as driven by the proposed self-organizational mechanism. ${ }^{21}$ In an acid solution (on the left branch of the dissolution curve), the dissolution rate of silica framework is higher than that for cation leaching, and as a result the material would dissolve congruently with no leached layer developed. As the $\mathrm{pH}$ of the solution increases, the dissolution rate becomes lower than the leaching rate, leading to the formation a leached surface layer. As the $\mathrm{pH}$ continuously rises, the system moves from the left branch to the right branch of the dissolution curve. When the dissolution rate becomes on the same order of magnitude as the mass exchange rate with the bulk solution, oscillatory dissolution may emerge. As the $\mathrm{pH}$ of the solution further increases, the dissolution rate eventually overtakes the mass exchange rate, leading to a "runaway" situation with a sharp increase in the cation concentration at the interface as well as in the dissolution rate. The sharp increase in both cation concentration and $\mathrm{pH}$ inevitably causes zeolite precipitation. $\Delta \mathrm{pH}=\mathrm{pH}-\mathrm{IEP}$, where IEP is the isoelectric point

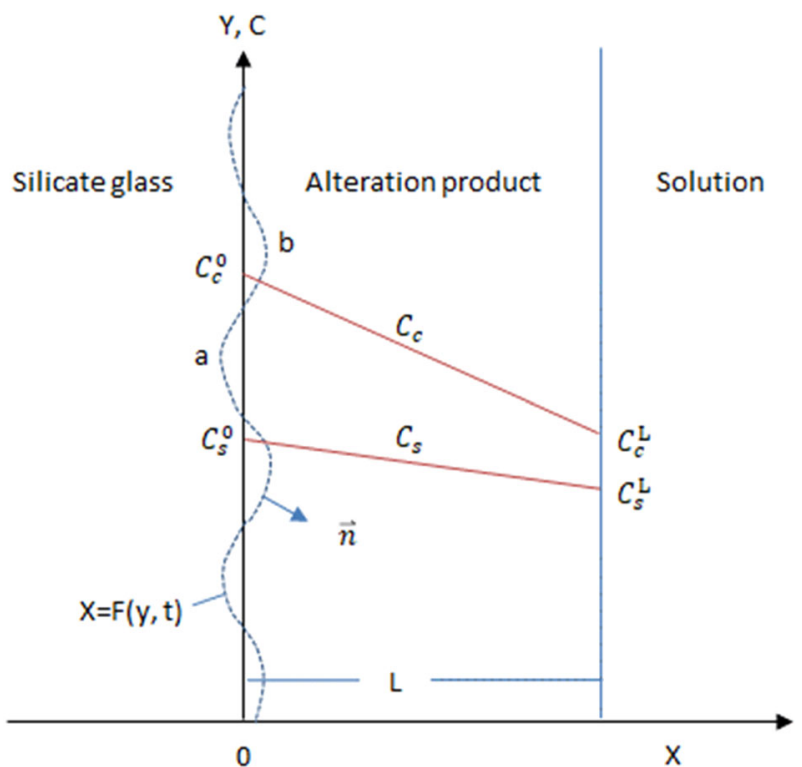

Fig. 3 Schematic representation of the system for the morphological instability analysis. Vertical axis represents both $Y$ coordinate and the concentrations of dissolved species

to lump model parameters to a minimum set of dimensionless parameters (see Methods):

$\theta=\frac{k L}{D_{s}} \quad \eta=\frac{C_{s}^{e}(\infty) D_{s}}{C_{\text {IEF }} D_{c}} \quad \epsilon=C_{s}^{e}(\infty) / \rho \quad \sigma=\frac{D_{s}}{D_{c}}$

where parameter $\theta$ characterizes the relative importance between the reaction rate and the diffusional flux; $\eta$ represents the diffusional flux ratio between dissolved $\mathrm{SiO}_{2}$ and cations; $\epsilon$ is a smallness parameter characterizing the disparity in molar density between solution and solid, and $\sigma$ is the ratio of diffusion coefficient between dissolved $\mathrm{SiO}_{2}$ and cations. Assume the solubility of silica framework on a planar surface, $C_{s}^{e}(\infty)$, to be represented by that of amorphous silica. ${ }^{32}$ The solubility of amorphous silica at $25^{\circ} \mathrm{C}$ and neutral pH is $10^{-2.6} \mathrm{M}$ (ref. ${ }^{33}$, Fig. 1). This solubility increases with both temperature and $\mathrm{pH}$ and could reach $\sim 0.5 \mathrm{M}$ at $\mathrm{pH} 10.5$ and $90^{\circ} \mathrm{C}$ (ref. ${ }^{33}$, fig. 9). Given the density $\left(2.52 \mathrm{~g} / \mathrm{cm}^{3}\right)$ of international simple glass and the weight percentage of $\mathrm{SiO}_{2}(56.2 \%)$ in the glass, ${ }^{15} \rho$ is estimated to be $24 \mathrm{~mol} \mathrm{SiO}_{2} / \mathrm{dm}^{3}$ glass. Considering the self-accelerating mechanism proposed above to be limited to the alkaline branch of the dissolution curve (Fig. 2), smallness parameter $\epsilon$ is therefore estimated to range approximately from $10^{-4}$ to $10^{-2}$.

In the scaling analysis, we treat the thickness of the alteration zone $(L)$ as a constant. In actual case, $L$ evolves as a material degrades, depending on the relative rates of alteration product removal at the outer rim of alteration products and the dissolution of pristine silicate material at the inner rim. The rate of the former highly depends on specific experimental or environmental conditions. Mathematically, a linear stability analysis would be made much easier if it is performed around a steady state. Therefore, to facilitate the analysis, we treat our system as if it reaches a steady state, called a quasi-steady state, as the thickness of the alteration zone evolves. In other words, we treat $L$ as a model parameter instead of a variable and then study the behavior of the system around a neighborhood of a given $L$ value.

A linear stability analysis of scaled equations (2) through (13) was performed (see Methods). In the analysis, we first introduce a perturbation of non-dimensional wave number $\omega$ to an initially planar reaction front and then determine the growth rate $(\zeta)$ of the perturbation as a function of wave number (see Methods):

$$
\zeta=\frac{\left.\omega^{3} \gamma \frac{\partial \bar{r}}{\partial \bar{u}}\right|_{s=0}-\left.\omega \frac{d \bar{r}}{d s}\right|_{s=0}}{\omega+\left.a_{c} \theta \eta \frac{\partial \bar{r}}{\partial \bar{v}}\right|_{s=0}+\left.a_{s} \theta \frac{\partial \bar{r}}{\partial \bar{u}}\right|_{s=0}}
$$

where $s=x-f(y, \tau) ; \bar{r}, \bar{u}$ and $\bar{v}$ are the scaled reaction rate and concentrations of dissolved $\mathrm{SiO}_{2}$ and cations, respectively, all evaluated at the planar dissolution front (as indicated by the overbars). Note that all three derivatives in equation (16) depend on the concentrations and the concentration gradients of dissolved $\mathrm{SiO}_{2}$ and cations at the surface. Therefore, as shown in equation (16), the stability of the dissolution front is controlled by (1) $\gamma, \theta, a_{c}$ and $a_{s,}$ and (2) the concentrations and the concentration gradients of dissolved species at the front. For a silicate glass, assuming the thickness of an alteration zone $(L)$ to be about $1 \mathrm{~mm}$, and using the surface energy $\left(E_{s}\right)$ of $\sim 4 \mathrm{~J} / \mathrm{m}^{2}$ (ref. ${ }^{34}$ ) $\left(=10^{-7} \mathrm{~m}\right), \gamma$ is estimated to be $\sim 10^{-4}$, based on the relationship of $\Gamma=2 v_{m} E_{s} / R_{g} T_{k}$ (see footnote 3 in ref. ${ }^{31}$ ), where $v_{m}$ is the molar volume of silica $\left(24 \mathrm{~cm}^{3} / \mathrm{mol}\right), R_{g}$ is the gas constant $(8.314 \mathrm{~J} / \mathrm{mol} /$ $\mathrm{K})$ and $T_{k}$ is the absolute temperature $(298 \mathrm{~K})$. A typical growth rate $\zeta$ of perturbation as a function of wave number $\omega$ is shown in Fig. 4a. Over a limited range of wave number, the growth rate of perturbation becomes positive, implying that the perturbations with these wave numbers would be amplified and the dissolution front then becomes unstable. The wave number $\omega_{\max }$ with a maximum growth rate determines the wave length of an actual pattern formed. The wave length $\left(2 \pi L / \omega_{\max }\right)$ is estimated to range from a few to hundreds of micrometers, consistent with observations ${ }^{17-19,23-25,35}$. Thus, our model provides a natural explanation for the formation of a highly wavy dissolution front as shown in Fig. 1 a.

The behavior of $\zeta(\omega)$ is, to a large extent, controlled by two terms in the numerator of equation (16). Since, according to Equation (24), $\left.\frac{\partial \bar{r}}{\partial \bar{u}}\right|_{s=0}=-\left(1+\beta \bar{v}^{m}\right)$ and is always negative, to make $\zeta$ positive, from Equation (23), it is required that

$$
\begin{aligned}
\left.\frac{d \bar{r}}{d s}\right|_{s=0} & =\left.\left.\frac{\partial \bar{r}}{\partial \bar{v}}\right|_{s=0} \frac{\partial \bar{v}}{\partial s}\right|_{s=0}+\left.\left.\frac{\partial \bar{r}}{\partial \bar{u}}\right|_{s=0} \frac{\partial \bar{u}}{\partial s}\right|_{s=0} \\
& =\left.m \beta \bar{v}^{m-1}(1-\bar{u}) \frac{\partial \bar{v}}{\partial s}\right|_{s=0}-\left.\left(1+\beta \bar{v}^{m}\right) \frac{\partial \bar{u}}{\partial s}\right|_{s=0}<0 .
\end{aligned}
$$


A
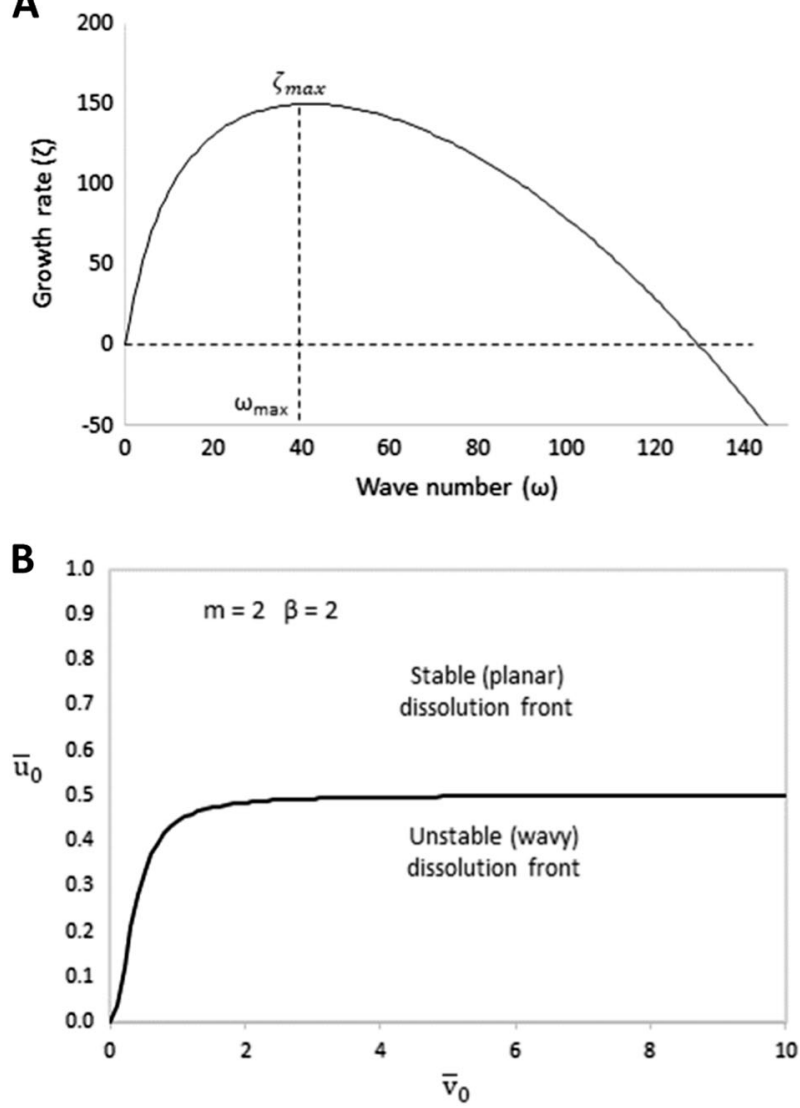

Fig. 4 Linear stability analysis. a Growth rate of a perturbation to a planar dissolution surface as a function of perturbation wave number $\omega(=2 \pi \mathrm{L} /$ wavelength $)$. A positive growth rate of a perturbation implies that the perturbation would be amplified and the dissolution front then becomes unstable. The wave number $\omega_{\max }$ with a maximum growth rate determines the wave length of an actual pattern formed. $\bar{u}_{0}=0.1, \bar{v}_{0}=8$ (ref. $^{21}$ ), $\gamma=10^{-4}$ (see the discussion in the text), $a_{s}=0.05, a_{c}=0.33, \theta=1, \eta=2, \beta=2$, and $m$ $=2\left(\right.$ ref. $\left.^{21}\right)$. $\mathbf{b}$ Behavior diagram delineating morphological instability in the parameter space of scaled silica and cation concentrations $\bar{u}_{0}$ and $\bar{v}_{0}$. A high cation concentration combined with a low $\mathrm{SiO}_{2}(\mathrm{aq})$ concentration favors morphological instability. For simplicity, the concentrations outside the corrosion product layer are here assumed to be zero

This is a necessary condition for morphological instability of aqueous dissolution of a silicate material. In other words, a dissolution rate gradient at the front $\left.\frac{d \vec{r}}{d s}\right|_{s=0}$ is a driving force for morphological instability. A more negative gradient $\left.\frac{d r}{d s}\right|_{s=0}$ would lead to a higher tendency for instability (larger $\zeta$ ) and consequently a shorter wavelength for pattern formation (larger w) (e.g., more compacted pitting spacing). Note that this negative rate gradient is solely contributed by a negative gradient of cation concentration, $\left.\frac{\partial \bar{v}}{\partial s}\right|_{s=0}$. In contrast, as shown in equation (17), the silica concentration gradient tends to stabilize a planar front (leads to negative ל). For fixed concentrations of dissolved species outside the alteration zone, the necessary condition depends only on the concentration of the species at the front: $\left.\frac{\partial \bar{u}}{\partial s}\right|_{s=0} \approx u_{1}-\bar{u}_{0}$ and $\left.\frac{\partial \bar{v}}{\partial s}\right|_{s=0} \approx v_{1}-\bar{v}_{0}$. As shown in Fig. 4b, a high cation concentration combined with a low $\mathrm{SiO}_{2}(\mathrm{aq})$ concentration favors a morphological instability. As shown in our previous work, ${ }^{21}$ these concentrations may oscillate with time during material degradation, and so does the wavelength of the front. In constructing Fig. $4 \mathrm{~b}$, due to the existence of dual time scales as discussed below, we assume that the steady state concentrations can be approximated linearly across the alteration zone (Fig. 3).
Interactions of morphological instability with oscillatory dissolution

In scaling equations (2) through (13), we constrain a typical time scale $(T)$ for morphological instability of a dissolution front such that a significant growth of a perturbation to the front would be observable [see equation (14) and Methods]. By a similar argument, to observe a significant variation in a dissolved concentration, from equations (19) through (20), the relevant time scale must be chosen to be $\sim 2 \epsilon T(=2 L / k)$ (see details in Methods), which represents a typical time scale for repetitive banding in alteration products Since $\epsilon$ is small $\left(10^{-4}\right.$ to $\left.10^{-2}\right)$ as discussed above, to simplify mathematical manipulations, as a first order approximation, the concentrations of dissolved silica and cations at a dissolution front, $\bar{u}_{0}$ and $\bar{v}_{0}$, in equations (19) and (20) can be treated as steady state variables in the analysis of morphological instability, i.e., $\epsilon \rightarrow 0$. As shown previously, ${ }^{21}$ these concentrations can oscillate with time, leading to repetitive bandings in alteration products as generally observed. ${ }^{25}$ Since the morphological instability depends on $\bar{u}_{0}$ and $\bar{v}_{0}$ as the concentrations oscillate at the dissolution front, the wavelength of morphological instability of the front would also oscillate.

As mentioned earlier, in an actual system, a dissolution front is constantly subjected to environmental perturbations. The onset of morphological instability can start at any time once the conditions for the instability are met. A stagnant solution condition, a high cation content in glass and the formation of a lower permeability alteration layer would all promote morphological instability of a glass dissolution front by enhancing the negative cation concentration gradient at the front. Under certain conditions, the onset of morphological instability could happen at an early time, even at time zero probably, of a dissolution process. Whether a perturbation can develop into an observable wavy front depends the relative rates of perturbation growth and dissolution front advancement. Let's denote the maximum growth rate of perturbation by $\zeta_{\max }$, i.e., $\zeta_{\max }=\zeta\left(\omega_{\max }\right)$. By trying different combinations of dissolved $\mathrm{SiO}_{2}$ and cation concentrations $\left(\bar{u}_{0}\right.$, $\bar{v}_{0}$ ) at a planar dissolution front, we found that $\zeta_{\max }$ could vary up to 150 (Fig. 4a). A typical time scale for perturbation growth is estimated more accurately to be $T / \zeta_{\max }=T \prime /\left(2 \zeta_{\max } \epsilon\right)$, where $T$ is a typical time scale for oscillatory dissolution and banding [see Eq. (37) in Methods]. Given $\epsilon=10^{-4}-10^{-2}$, we postulate that the growth rate of a morphological perturbation could be small compared to the dissolution front advancement but, in some cases, it can become comparable with the later. For illustration, assume that the morphological instability started at time zero of the dissolution for the sample shown in Fig. 1a. As the dissolution front advanced, the perturbation of morphological instability became amplified and developed into a wavy front as we see now in the figure. As shown in the figure, over 55 days, an alteration rim of $\sim 660 \mu \mathrm{m}$ thick was developed. The corrosion rate is thus estimated to be $\sim 11 \mu \mathrm{m} /$ day, which is high as compared to the initial rate of the international simple glass generally reported $\left(\sim 1.5 \mu \mathrm{m} /\right.$ day ref. $\left.^{36}\right)$. This high rate could be attributed to a local accumulation of the cations released from glass dissolution as our model suggests. Given the amplitude of the wavy front $(50-100 \mu \mathrm{m})$ (Fig. 1a), we estimate the growth rate of morphological instability to be approximately $1 \mu \mathrm{m} /$ day, 10 times slower than the rate of dissolution front advancement, which seems consistent with our theoretical estimation.

Consequently, there are four possibilities for the interactions of morphological instability with oscillatory dissolution and therefore for pattern formations. In case 1, the growth of morphological perturbations is much slower than dissolution front advancement or the aqueous species concentrations oscillate within a morphologically stable field (Fig. $5 \mathrm{a}$ ). In this case, only repetitive planar bandings are produced in the alteration zone. In case 2 , the growth rate of morphological perturbations is comparable with 
A

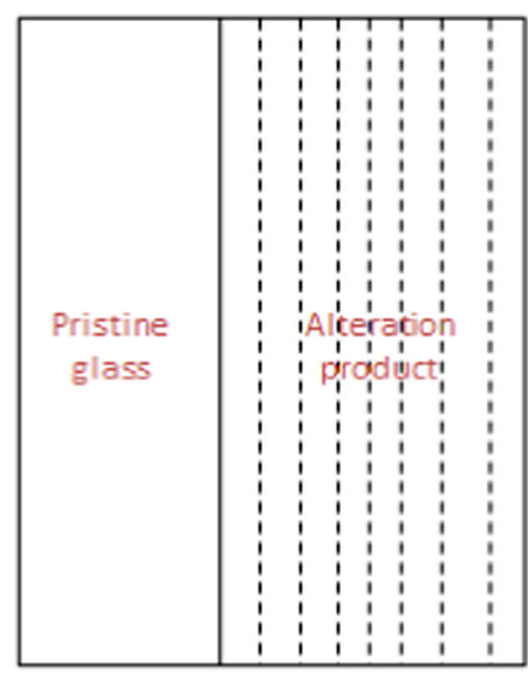

C

Incoherent wavy banding

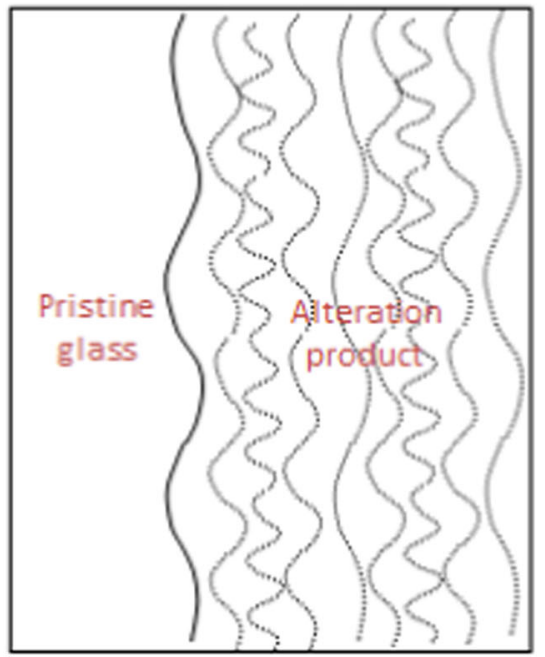

B

Coherent wavy banding

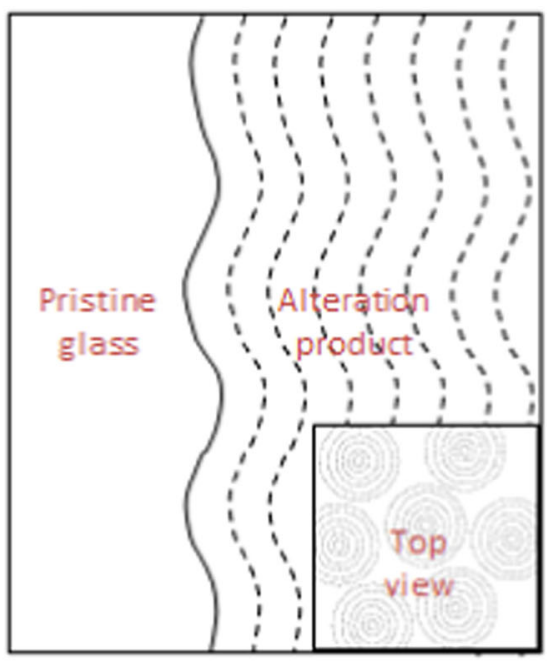

D

Wawy front w/o banding

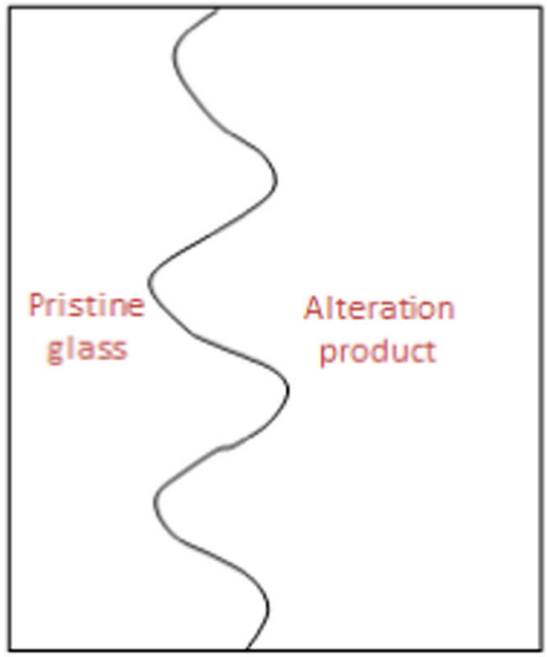

Fig. 5 Interactions of morphological instability with oscillatory dissolution of silicate materials. a Formation of coherent wavy bands in a case where the growth of morphological perturbations is much slower than dissolution front advancement or the aqueous species concentrations oscillate within a morphologically stable field. b Formation of coherent wavy bands in a case where the growth rate of morphological perturbations is comparable with the rate of dissolution front advancement and the concentration oscillations excursion into a morphologically unstable field with narrowly ranged wavelengths. c Formation of incoherent alteration bands in a case where the growth rate of morphological perturbations is comparable with the rate of dissolution front advancement and the concentration oscillations cross over a morphologically unstable field with a wide range of wavelengths. $\mathbf{d}$ Formation of a pattern with no banding but a wavy dissolution front in a case where a dissolution front may be morphologically unstable but not oscillatory

the rate of dissolution front advancement and the concentration oscillations excursion into a morphologically unstable field with narrowly ranged wavelengths, resulting in a set of coherent wavy bands (Figs. $1 \mathrm{~b}$ and $5 \mathrm{~b}$ ). On a view plane parallel to the dissolution front, these wavy bandings can be expressed as concentric growth rings (ref. ${ }^{23}$, fig. 7b; fig. 5b, insert). Obviously, the concentric bands formed as such would not follow a banding spacing law for typical Liesegang rings as observed. ${ }^{25}$ In case 3 , the growth rate of morphological perturbations is comparable with the rate of dissolution front advancement and the concentration oscillations cross over a morphologically unstable field with a wide range of wavelengths, leading to a set of incoherent alteration bands (Fig. $5 c)$. The structure within each band could be relatively messy or chaotic, due to a successive juxtaposition of incremental alteration layers with distinct wavelengths (Figs. 1c, d). Finally, since oscillatory dissolution occurs in a limited parameter space (ref. ${ }^{21}$, fig. 4), in case 4, a dissolution front may be morphologically unstable but not oscillatory, which would lead to a pattern with no banding but a wavy dissolution front (Fig. 5d), as shown in Fig. 1a, if the growth rate of morphological perturbations is comparable with the rate of dissolution front advancement. Depending on the initial and boundary conditions imposed, material dissolution can shift from one regime to another as the dissolution proceeds. For example, as we have shown in our earlier work, ${ }^{21}$ at the beginning of the dissolution, no alteration zone is developed and the dissolution process is overwhelmed by diffusion, resulting in a plain alteration zone with no oscillations. As the alteration zone builds up, the dissolution rate becomes comparable with the diffusion rate, and oscillatory dissolution emerges (ref. ${ }^{21}$, fig. 4A). Since, as mentioned earlier, morphological instability could happen at an early time of a dissolution process. As a result, we would see a transition from a wavy dissolution front with no 
banding to a wavy front with bandings, as shown in Fig. 1b. Since both solution chemistry and mineral precipitation rate vary at a dissolution front, the patterns formed in an alteration zone can be expressed as either chemical or structural contrasts. ${ }^{23,25,35}$ Therefore, the self-accelerating mechanism we proposed can provide a systematical prediction of a whole suite of pattern formations observed in silicate material degradation ${ }^{23-25,37}$ (Fig. 1).

\section{DISCUSSIONS}

In silicate glass dissolution experiments, a gap up to $50 \mu \mathrm{m}$ wide was observed between an alteration zone and a neighboring pristine glass, which was interpreted as an indicator of a former atomically sharp reaction interface. ${ }^{23}$ The development of this gap could potentially affect the concentration gradients of dissolved species at the dissolution front. Let's consider the cation concentration gradient, the main driving force for the morphological instability of a silicate material dissolution front. Based on a mass-continuity argument (i.e., the flux remaining the same in the existence of a gap or no gap), we have:

$$
\frac{\left(\left.\frac{\partial \bar{v}}{\partial s}\right|_{s=0}\right)_{\text {gap }}}{\left(\left.\frac{\partial \bar{v}}{\partial s}\right|_{s=0}\right)_{\text {no gap }}}=\frac{D_{c}(\phi)}{D_{c}^{0}} \approx \phi^{2}
$$

where $D_{c}^{0}$ is the diffusion coefficient of cations in bulk water, and $\phi$ is the porosity of the alteration products. It is assumed that the tortuosity of the alteration products to be $\sim 1 / \phi$ (ref. $^{38}$ ). Assuming $\phi=0.1$, Equation (18) shows that the concentration gradient of cations at the front could be reduced by 100 times if a gap is present, implying that its presence would significantly enhance the stability of the front, consistent with experimental observations. ${ }^{23}$ Backscattered electron images show that such gaps were developed in the alteration of borosilicate glasses, and the repetitive alteration bands become less wavy from the outer rim of the alteration zone to the dissolution surface. ${ }^{23}$

The work presented here highlights the importance of local solution chemistry in the alteration of geological materials. ${ }^{39}$ Water at a reaction front is generally stagnant and its chemistry could significantly deviate from that of advective pore water. Using the chemistry of advective pore water to predict a weathering rate, as usually done, may significantly underestimate or overestimate the reaction rate. The fast carbon sequestration rate observed in the injection of $\mathrm{CO}_{2}$ into a basaltic rock ${ }^{5}$ may possibly be attributed to the accelerated silicate dissolution due to a local cation accumulation at a dissolution front. Furthermore, reactive surface area of a geological material is an important, but the least constrained, parameter for geochemical modeling. The morphological instability discussed above may add another level of complexity to the quantification of reactive surface area in actual geochemical systems. Due to the morphological instability, the reactive surface area may increase as the reaction proceeds. This is an important factor that needs to be considered in a longterm performance assessment of a silicate material as a waste form for nuclear waste disposal. Depending on experimental conditions, in principle, a local concentration variation could propagate through the alteration layer and therefore could be detected in the bulk solution outside the alteration layer, though the magnitude of the variation in the bulk solution could be much smaller than that at the dissolution front. As a matter of fact, temporal concentration oscillations were observed in the bulk solution in silicate mineral dissolution experiments. ${ }^{40}$

In addition, the proposed morphological instability may offer a sensible explanation for many other textural observations of material degradation in both natural and engineered systems. It is commonly observed that a chemical weathering front can deeply pit into a silicate mineral grain, such as a feldspar crystal, during mineral alteration. ${ }^{41,42}$ Similarly, a hemispherical-shape alteration front was observed deeply penetrating into a pristine glass domain. ${ }^{24}$ Such textures could be a manifestation of the proposed morphological instability-a localized concentration of cations released from an alteration reaction would further accelerate the reaction. Traditionally, pitting on a mineral surface has been attributed to the preferential dissolution on structural defects. ${ }^{43} \mathrm{~A}$ problem with this assumption is that the observed reaction front pitting and penetration are generally beyond a typical spatial correlation scale of mineral structural defects. It is particularly problematic for silicate glasses in which no long-range structural correlation exists but there are still $\mu \mathrm{m}$-scale pits developed on a dissolution surface (ref. ${ }^{23}$, fig. 5). Therefore, there must be other mechanism in driving mineral dissolution and pitting. Our work suggests that structural defects may play a role as an initial perturbation to a dissolution front but a long-range pitting or a localized reaction front penetration is likely to be driven by the proposed morphological instability. Furthermore, it would be expected that a glass with a higher cation content is more likely to have pitting dissolution. In contrast, since release of borate would counter the $\mathrm{pH}$ rise by cations, a glass containing a borate component may stabilize a dissolution front, thus reducing the likelihood for pitting. Therefore, the advancement rate and the morphology of a dissolution front could be engineeringly controlled, if needed, by adjusting glass compositions.

As mentioned earlier, there are two schools of thought on the actual mechanism of silicate material degradation: the surface layer concept ${ }^{12-14}$ and the direct dissolution-precipitation concept. ${ }^{17-20}$ The work presented here is not intended to make a judgement one vs. another on the two proposed concepts. As a matter of fact, our model seems general enough to apply to both situations. The point we want to make here is that the complex behaviors observed in silicate material degradation, including the occurrence of a sharp dissolution front or a leached surface layer, are likely to be the manifestations of a single unified mechanism under different circumstances. We have shown that the selfaccelerating mechanism we have proposed can provide a consistent explanation for many, if not all, of the key features of the observed silicate material corrosion phenomena. We would also like to point out that the model analysis presented here, together with our earlier work, ${ }^{21}$ is intended to set up a theoretical framework for unraveling complex nonlinear dynamics of silicate material degradation. A full dynamics analysis of the system requires a numerical solution of equations (2) through (13), with a specified evolution of alteration layer thickness $L$, on two different time scales for banding and morphological instability. While a linear stability analysis presented here helps clarify the types of dissolution patterns that would form and the conditions for their formation, a full dynamics analysis will provide more detail information about a possible transition from one dissolution pattern to another in a specific dissolution experiment or process. The results of a full dynamics analysis of our model will be presented elsewhere.

\section{METHODS}

Scaling

Using scaling factors introduced in equations (14), equations (2) through (13) can be cast into:

Within $f(y, \tau)<x<1$ :

$\epsilon \theta \frac{\partial u}{\partial \tau}=\nabla^{2} u$

$\epsilon \theta \sigma \frac{\partial v}{\partial \tau}=\nabla^{2} v$ 


$$
\begin{aligned}
& \text { At } x=f(y, \tau): \\
& a_{s} \theta r=-\vec{\nabla} u \cdot \vec{n} \\
& a_{c} \theta \eta r=-\vec{\nabla} v \cdot \vec{n} \\
& r=\left(1+\beta v^{m}\right)(1+\gamma \kappa-u) \\
& \vec{r}=\left(1+\beta \bar{v}^{m}\right)(1-\bar{u}) \\
& \kappa=-\frac{\partial^{2} f}{\partial y^{2}} /\left[1+\left(\frac{\partial f}{\partial y}\right)^{2}\right]^{3 / 2} \\
& \vec{n}=\left(1,-\frac{\partial f}{\partial y}\right)^{T} /\left[1+\left(\frac{\partial f}{\partial y}\right)^{2}\right]^{1 / 2} \\
& \frac{\partial f}{\partial \tau}=\bar{r}-r\left[1+\left(\frac{\partial f}{\partial y}\right)^{2}\right]^{1 / 2} \\
& \text { At } x=1: \\
& u=u_{1} \quad v=v_{1} .
\end{aligned}
$$

Repetitive banding: planar-front solution

For a planar dissolution front $(f=0)$, equations (19) through (28) are reduced to:

$$
\begin{aligned}
& \epsilon \theta \frac{\partial \bar{u}}{\partial \tau}=\frac{\partial^{2} \bar{u}}{\partial x^{2}} \\
& \epsilon \theta \sigma \frac{\partial \bar{v}}{\partial \tau}=\frac{\partial^{2} \bar{v}}{\partial x^{2}}
\end{aligned}
$$

$$
\text { at } x=0 \text { : }
$$

$a_{s} \theta \bar{r}=-\frac{\partial \bar{u}}{\partial x}$

$a_{c} \theta \eta \bar{r}=-\frac{\partial \bar{v}}{\partial x}$

$\bar{r}=\left(1+\beta \bar{v}^{m}\right)(1-\bar{u})$

$$
\text { at } x=1 \text { : }
$$

$\bar{u}=u_{1}$ and $\bar{v}=v_{1}$.

where $\bar{u}, \bar{v}$ and $\bar{r}$ are the $u, v$, and $r$ referred to the planar front. By integrating equations (29) and (30) over the alteration layer and assuming the concentrations of dissolved silica and cations can be approximated linearly within the alteration product layer $(0 \leq x \leq 1)$ (ref. $\left.{ }^{31}\right)$, we obtain:

$\theta \frac{d \bar{u}_{0}}{d \tau \prime}=a_{s} \theta\left(1+\beta \bar{v}_{0}^{m}\right)\left(1-\bar{u}_{0}\right)+u_{1}-\bar{u}_{0}$

$\theta \sigma \frac{d \bar{v}_{0}}{d \tau \prime}=a_{s} \theta\left(1+\beta \bar{v}_{0}^{m}\right)\left(1-\bar{u}_{0}\right)+v_{1}-\bar{v}_{0}$

where

$\tau^{\prime}=t / T /$ and $T \prime=2 \epsilon T=2 L / k$

and $\bar{u}_{0}$ and $\bar{v}_{0}$ represent the $\bar{u}$ and $\bar{v}$ evaluated at the front. The new time scale $\left(T^{\prime}\right)$ is chosen such that a significant variation in a dissolved concentration can be observed. As shown in reference, ${ }^{21}$ equations (35) and (36) can have oscillatory solutions, which are postulated to be responsible for the formation of repetitive bands of alteration products. Therefore, $T$ is the time scale for material dissolution and banding.

\section{Linearization}

Let $\epsilon \rightarrow 0$, and also make a coordinate transformation of $s=x-f(y, \tau)$. Equations (19) to (28) can then be linearized by introducing a perturbation around a steady state planar solution $(\bar{u}, \bar{v}, \bar{r})^{31}$ :

$u=\bar{u}+\delta u \quad v=\bar{v}+\delta v \quad f=\delta f \quad r=\bar{r}+\delta r \quad \kappa=\delta \kappa$

We obtain the following linearized equations:
Within $0<s<1$

$$
\frac{\partial^{2} \delta u}{\partial s^{2}}+\frac{\partial^{2} \delta u}{\partial y^{2}}-\frac{\partial \bar{u}}{\partial s} \frac{\partial^{2} \delta f}{\partial y^{2}}=0
$$

$\frac{\partial^{2} \delta v}{\partial s^{2}}+\frac{\partial^{2} \delta v}{\partial y^{2}}-\frac{\partial \bar{v}}{\partial s} \frac{\partial^{2} \delta f}{\partial y^{2}}=0$

At $s=0$ :

$\delta r=-\left(1+\beta \bar{v}_{0}^{m}\right)\left(\gamma \frac{\partial^{2} \delta f}{\partial y^{2}}+\delta u\right)+m \beta \bar{v}_{0}^{m-1}\left(1-\bar{u}_{0}\right) \delta v$

$a_{s} \theta \delta r=-\frac{\partial \delta u}{\partial s}$

$a_{s} \theta \eta \delta r=-\frac{\partial \delta v}{\partial s}$

$\frac{\partial \delta f}{\partial \tau}=-\delta r$

At $s=1$ :

$\delta u=\frac{\partial \bar{u}}{\partial s} \delta f \quad \delta v=\frac{\partial \bar{v}}{\partial s} \delta f$

where $\bar{u}_{0}$ and $\bar{v}_{0}$ are the steady state dissolved $\mathrm{SiO}_{2}$ and cation concentrations at a planar dissolution front.

Here we do not solve the steady state concentrations directly for a planar front. As discussed above (see a detail treatment in ref. ${ }^{31}$ ), due to the existence of dual time scales, the concentrations calculated from the earlier model for oscillatory dissolution ${ }^{21}$ can be treated as the steady state concentrations for the morphological instability analysis. For simplicity, we further assume that the steady state concentrations can be approximated linearly across the alteration zone (Fig. 3).

\section{Dispersion equation}

Assume that the linearized equations have a solution in the form of:

$$
\begin{aligned}
\delta u & =\hat{u}(s) e^{\zeta \tau} \cos (\omega y) \\
\delta v & =\hat{v}(s) e^{\zeta \tau} \cos (\omega y) \\
\delta f & =\hat{f} e^{\zeta \tau} \cos (\omega y) \\
\delta r & =\hat{r} e^{\zeta \tau} \cos (\omega y)
\end{aligned}
$$

where $\hat{u}(s), \hat{v}(s), \hat{f}$ and $\hat{r}$ are the infinitesimal amplitudes of perturbations $\delta u, \delta v, \delta f$, and $\delta r$ and independent of $y$ and $\tau$. $\hat{f}$ and $\hat{r}$ are also independent of $s$. We now have: Within $0<s<1$ :

$\frac{d^{2} \hat{u}}{d s^{2}}-\omega^{2} \hat{u}+\omega^{2} \frac{\partial \bar{u}}{\partial s} \hat{f}=0$

$\frac{d^{2} \hat{v}}{d s^{2}}-\omega^{2} \hat{v}+\omega^{2} \frac{\partial \bar{v}}{\partial s} \hat{f}=0$

At $s=0$ :

$\hat{r}=-\left(1+\beta \bar{v}_{0}^{m}\right)\left(-\gamma \omega^{2} \hat{f}+\hat{u}\right)+m \beta \bar{v}_{0}^{m-1}\left(1-\bar{u}_{0}\right) \hat{v}$

$a_{s} \theta \hat{r}=-\frac{d \hat{u}}{d s}$

$a_{s} \theta \eta \hat{r}=-\frac{d \hat{v}}{d s}$

$\zeta \hat{f}=-\hat{r}$

At $s=1$ :

$\hat{u}=\frac{\partial \bar{u}}{\partial s} \hat{f} \quad \hat{v}=\frac{\partial \bar{v}}{\partial s} \hat{f}$

Equations (47 and 48 ) have a solution in the form of:

$\hat{u}(s)=d_{11} e^{\omega s}+d_{12} e^{-\omega s}+\frac{\partial \bar{u}}{\partial s} \hat{f}$

$\hat{v}(s)=d_{21} e^{\omega s}+d_{22} e^{-\omega s}+\frac{\partial \bar{v}}{\partial s} \hat{f}$

where $d_{i j}$ is a constant. Inserting equations (54-55) into the boundary 
conditions (49-53), we obtain a linear set of equations with respect to $\hat{u}(0)$, $\hat{v}(0)$ and:

$$
\begin{aligned}
& \hat{u}(0)-\left[\frac{a_{s} \theta \zeta\left(e^{2 \omega}-1\right)}{\omega\left(e^{2 \omega}+1\right)}+\left.\frac{\partial \bar{u}}{\partial s}\right|_{s=0}\right] \hat{f}=0 \\
& \hat{v}(0)-\left[\frac{a_{s} \theta \zeta\left(e^{2 \omega}-1\right)}{\omega\left(e^{2 \omega}+1\right)}+\left.\frac{\partial \bar{v}}{\partial s}\right|_{s=0}\right] \hat{f}=0
\end{aligned}
$$

$\zeta \hat{f}-\left(1+\beta \bar{v}_{0}^{m}\right)\left(-\gamma \omega^{2} \hat{f}+\hat{u}\right)+m \beta \bar{v}_{0}^{m-1}\left(1-\bar{u}_{0}\right) \hat{v}=0$

For equations (56-58) to have a nontrivial solution, the determinant of the coefficient matrix must be zero:

$$
\left|\begin{array}{ccc}
1 & 0 & -\frac{a_{s} \theta \zeta\left(e^{2 \omega}-1\right)}{\omega\left(e^{2 \omega}-1\right)}-\left.\frac{\partial \bar{u}}{\partial s}\right|_{s=0} \\
0 & 1 & -\frac{a_{s} \theta \zeta\left(e^{2 \omega}-1\right)}{\omega\left(e^{2 \omega}-1\right)}-\left.\frac{\partial \bar{v}}{\partial s}\right|_{s=0} \\
-\left(1+\beta \bar{v}_{0}^{m}\right) & m \beta \bar{v}_{0}^{m-1}\left(1-\bar{u}_{0}\right) & \zeta+\gamma \omega^{2}\left(1+\beta \bar{v}_{0}^{m}\right)
\end{array}\right|=0
$$

From equation (59), we obtain:

$$
\zeta=\frac{\left.\omega^{3} \gamma \frac{\partial \bar{r}}{\partial \bar{u}}\right|_{s=0}-\left.\omega \frac{d \bar{r}}{d s}\right|_{s=0}}{\omega+\left.a_{c} \theta \eta \frac{e^{2 \omega}-1}{e^{2 \omega}+1} \frac{\partial \bar{r}}{\partial \bar{v}}\right|_{s=0}+\left.a_{s} \theta \frac{e^{2 \omega}-1}{e^{2 \omega}+1} \frac{\partial \bar{r}}{\partial \bar{u}}\right|_{s=0}}
$$

A planar front corresponds to $\omega=0$. To observe a morphological instability, the wavelength of a wavy front must be smaller than the typical scale of a system, that is, $\frac{2 \pi}{\omega}<1$. Since $\omega>2 \pi, \frac{e^{2 \omega}-1}{e^{2 \omega}+1} \sim 1$, and we then obtain equation (16).

\section{DATA AVAILABILITY}

The authors declare that the data supporting the findings of this study are available within the paper.

\section{ACKNOWLEDGEMENTS}

Sandia National Laboratories is a multi-mission laboratory managed and operated by National Technology and Engineering Solutions of Sandia, LLC., a wholly owned subsidiary of Honeywell International, Inc., for the U.S. Department of Energy's National Nuclear Security Administration under contract DE-NA-0003525. The work was supported by DOE Spent Fuel Waste Science \& Technology (SFWST) Program.

\section{AUTHOR CONTRIBUTIONS}

Y.W. developed both the conceptual and the mathematical models. C.F.J collected literature data on model parameters. C.L. and J.I. collected and provided images of pattern formations in glass dissolution. Y.W. wrote the first draft of the manuscript, and K.L.K made extensive editing of the first draft. All five authors discussed the results and commented on the manuscript.

\section{ADDITIONAL INFORMATION}

Competing interests: The authors declare no competing interests.

Publisher's note: Springer Nature remains neutral with regard to jurisdictional claims in published maps and institutional affiliations.

\section{REFERENCES}

1. Berner, R. A. \& Kothavala, Z. GEOCARB III: a revised model of atmospheric $\mathrm{CO}_{2}$ over Phanerozoic time. Am. J. Sci. 301, 182-204 (2001).

2. Brady, P. V. The effect of silicate weathering on global temperature and atmospheric $\mathrm{CO}_{2}$. J. Geophys. Lett. 96, 18101-18106 (1991).

3. White, A. F. \& Brantley, S. L. The effect of time on the weathering of silicate minerals: why do weathering rates differ in the laboratory and field? Chem. Geol. 2002, 479-506 (2003).

4. Matter, J. M. \& Kelemen, P. B. Permanent storage of carbon dioxide in geological reservoirs by mineral carbonation. Nat. Geosci. 2, 837-841 (2009).

5. Matter, J. M. et al. Rapid carbon mineralization for permanent disposal of anthropogenic carbon dioxide emissions. Science 352, 1312-1414 (2016).

6. Kulprathipanja, S. Zeolites in Industrial Separation and Catalysis. (Wiley-VCH, Weinheim, 2010)

7. Murata, H. Handbook of Optical Fibers and Cables. (Marcel Dekker, New York, 1996).
8. Hin, T. S. Engineering Materials for Biomedical Applications. (World Scientific Publishing Co, New Jersey, 2004).

9. Domone, P. \& Illston, J. Construction Materials. (Spon Press, New York, 2010).

10. Cailleteau, C. et al. Insight into silicate-glass corrosion mechanisms. Nat. Mater. 7, 978-983 (2008).

11. Gin, S. Open scientific questions about nuclear glass corrosion. Procedia Mater. Sci. 7, 163-171 (2014).

12. Dran, J.-C., Petit, J.-C. \& Brousse, C. Mechanism of aqueous dissolution of silicate glasses yield by fission tracks. Nature 319, 485-487 (1986).

13. Doremus, R. H. Interdiffusion of hydrogen and alkali ions in a glass surface. J. NonCryst. Solids 19, 137-144 (1975).

14. Petit, J. C., \& PaccagnellaA. et al. Hydrated-layer formation during dissolution of complex silicate glasses and minerals. Geochim. Cosmochim. Acta 54, 1941-1955 (1990).

15. Gin, S. et al. Origin and consequences of silicate glass passivation by surface layers. Nat. Commun. 6, 6360 (2015).

16. Gin, S. et al. Dynamics of self-reorganization explains passivation of silicate glass. Nat. Commun. 9, 2169 (2018).

17. Putnis, A. Sharpened interface. Nat. Mater. 14, 261-261 (2015).

18. Hellmann, R. et al. Nanometre-scale evidence for interfacial dissolutionreprecipitation control of silicate glass corrosion. Nat. Mater. 14, 307-311 (2015).

19. Ruiz-Agudo, E., Putnis, C. V., Rodriquez-Navarro, C. \& Putnis, A. Mechanism of leached layer formation during chemical weathering of silicate minerals. Geology 40, 947-950 (2012)

20. Geisler, T. et al. The mechanism of borosilicate glass corrosion revisited. Geochim. Cosmochim. Acta 158, 112-129 (2015).

21. Wang, Y., Jove-Colon, C. F. \& Kuhlman, K. L. Nonlinear dynamics and instability of aqueous dissolution of silicate glasses and minerals. Sci. Rep. 6, 30256 (2016).

22. Sekerka, R. F. Morphological instability. In Crystal Growth: An introduction (ed. Hartman, P.) 403-443 (North-Holland, Amsterdam, 1973).

23. Dohmen, L. et al. Pattern formation in silicate glass corrosion zones. Int. J. Appl. Glass Sci. 4, 357-370 (2013).

24. Silvestri, A., Molin, G. \& Salviulo, G. Archaeloogical glass alteration products in marine and land-based environments: morphological, chemical and microtextural characterization. J. Non-Cryst. Solids 351, 1338-1349 (2005).

25. Schalm, O. \& Anaf, W. Laminated altered layers in historical glass: density variations of silica nanoparticle random packings as explanation for the observed lamellae. J. Non-Cryst. Solids 442, 1-16 (2016).

26. Rozalén, M. L. et al. Experimental study of the effect of pH on the kinetics of montmorillonite dissolution at $25^{\circ} \mathrm{C}$. Geochim. Cosmochim. Acta 72, 4224-4253 (2008).

27. Skorina, T. \& Allanore, A. Aqueous alteration of potassium-bearing aluminosilicate minerals: from mechanism to processing. Green. Chem. 17, 2123-2136 (2015).

28. Wolery, W. G., Tester, J. W. \& Grigsby, C. O. Quartz dissolution kinetics from 100 $200^{\circ} \mathrm{C}$ as a function of $\mathrm{pH}$ and ionic strength. AlChE J. 42, 3442-3457 (1996).

29. Strachan, D. M. et al. The characterization and testing of candidate immobilization forms for the disposal of plutonium. In Waste Management'98 Conf. Proc. (Laser Options Inc., Tucson, AZ, 1998). ID 65-08, CDROM.

30. Rimstidt, J. D. Rate equations for sodium catalyzed quartz dissolution. Geochim Cosmochim. Acta 167, 195-204 (2015).

31. Wang, Y. \& Merino, E. Origin of fibrosity and banding in agates from flood basalts. Am. J. Sci. 295, 49-77 (1995).

32. Neeway, J. J., Ryan, J. V., Pierce, E. M., Qafoku, N. P., Freedman, V. L. A Strategy to Conduct An Analysis of the Long-term Performance of Low-activity Waste Glass in A Shallow Subsurface Disposal System at Hanford. (Pacific Northwest National Laboratory, 2014).

33. Eikenberg, J. On the Problem of Silica Solubility at High pH. (Paul Scherrer Institut, 1990).

34. Rimsza, J. M., Jones, R. E. \& Criscenti, L. J. Surface structure and stability of partially hydroxylated silica surfaces. Langmuir 22, 3882-3891 (2017).

35. Geisler, T. et al. Aqueous corrosion of borosilicate glass under acidic conditions: A new corrosion mechanism. J. Non-Cryst. Solids 356, 1458-1465 (2010).

36. Inagaki, Y., Kikunaga, T., Idemitsu, K. \& Arima, T. Initial dissolution rate of the international simple glass as a function of $\mathrm{pH}$ and temperature measured using microchannel flow-through test method. Int. J. Appl. Glass Sci. 4, 317-327 (2013).

37. Bianco, B. D., Bertoncello, R., Milanese, L. \& Barison, S. Glasses on the seabed: surface study of chemical corrosion in sunken Roman glasses. J. Non-Cryst. Solids 343, 91-100 (2004).

38. Oelkers, E. H. Physical and chemical properties of rocks and fluids for chemical mass transport calculations. Rev. Mineral. 34, 131-191 (1996)

39. Ruiz-Agudo, E., Rodriquez-Navarro, C. \& PutnisA, et al. Control of silicate weathering by interface-coupled dissolution-precipitation processes at the mineralsolution interface. Geology 77, 567-570 (2016). 
40. Falmon, J. Oscillatory silicon and aluminum aqueous concentrations during experimental aluminosilicate weathering. Geochim. Cosmochim. Acta 60, 2901-2907 (1996)

41. Velbel, M. A. Weathering of hornblende to ferruginous products by a dissolutionreprecipitation mechanism: petrography and stoichiometry. Clays Clay Miner. 37, 515-524 (1989).

42. Anand, R. R., Gilkes, R. J., Armitage, T. M. \& Hillyer, J. W. Feldspar weathering in lateritic saprolite. Clays Clay Miner. 33, 31-43 (1985).

43. Dove, M. P., Han, N. \& De Yoreo, J. J. Mechanism of classical crystal growth theory explain quartz and silicate dissolution behavior. PNAS 102, 15357-15362 (2005).

(i) Open Access This article is licensed under a Creative Commons

cc) Attribution 4.0 International License, which permits use, sharing,

adaptation, distribution and reproduction in any medium or format, as long as you give appropriate credit to the original author(s) and the source, provide a link to the Creative Commons license, and indicate if changes were made. The images or other third party material in this article are included in the article's Creative Commons license, unless indicated otherwise in a credit line to the material. If material is not included in the article's Creative Commons license and your intended use is not permitted by statutory regulation or exceeds the permitted use, you will need to obtain permission directly from the copyright holder. To view a copy of this license, visit http://creativecommons. org/licenses/by/4.0/.

(c) The Author(s) 2018 